\title{
From the journals
}

\section{Prevention of sports injuries in children}

Annually a large number of youth get injured while participating in sports. Although it is not possible to prevent all injuries, risk of injury can be minimised by following the simple instructions given below. (Preventive Medicine 2001; 31: 11-4).

* A pre-participation check will help detect medical conditions and discuss proper training technique and nutrition. 'Out-of-shape' kids are more likely to injure themselves than fit children.

* Wear protective equipment throughout play.

* Injured children should not return to play until after complete recovery. Those who are injured have a high risk of reinjury if they resume play before complete healing. Although children may be able to "play through" some minor injury, it can aggravate injury and cause extensive damage.

* Avoid vigorous exercise when the temperature is high. To prevent heat related injury on hot days, limit the activity to early morning or late afternoon. Make sure to consume adequate amounts of fluid. Children with fever or severe diarrhoea are more susceptible to dehydration and should avoid excess physical activity, especially when it is hot.

\section{Early mother-child intervention as a possible source of differential development of children at risk}

Understanding the causes for biologically vulnerable (eg low birth weight) and socially disadvantaged children to develop differently is essential in planning effective prevention and intervention programs. Early parenting has a decisive impact on the behavioural adjustment of at-risk children. Emotionally responsive and sensitive caregiving in early infancy can modify the effect of low birth weight on child adjustment up to school age, and protect against the adverse influences of family disadvantage. Early parenting is an effective intervention strategy for infants at risk. \{Development Medicine and Child Neurology 2001; 43: 293-300).

\section{Risk of death from neonatal meningitis}

A study over 11 years on neonatal meningitis is reported from South Africa. Out of 88 babies those with low birth weight (less than 1500 grams) and those who had elevated CSF protein had an increased risk of death. (Journal of Tropical Paediatrics 2000; 46: 237-9).

\section{Measles encephalopathy without classical features of measles}

A recent study from India has looked at the aetiology of encephalopathy in children aged 1 to 12 years. Of 28 specimens tested 17 had IgM antibodies to measles. A high isolation rate of measles virus from CSF and serum of patients, and the presence of IgM antibodies to measles proved the aetiology of the outbreak to be measles. Some notable features of these cases were the absence of the typical measles rash, non-clustering of cases and absence of detectable exposure to classical measles. (Indian Paediatrics 2001; 38: 589-95). It is possible that some of the cases of encephalopathy that we see here, but are unable to determine the aetiology, are due to measles.

\section{Asymptomatic amicrofilaraemics (endemic normals) - what happens to them?}

In filariasis endemic areas there is a well recognised group of people who are continuously exposed to the infection but remain apparently normal. They remain uninfected, symptom free and microfilariae negative. They are the endemic normals. Not much information is available about what happens to them in the long term. A longitudinal study from Orissa, India, reports on the progress of endemic normals from two villages who were followed up for 10 and 6 years. This study has shown that most endemic normals remain that way (over 10 years) and that a few men developed hydrocoele. Conversion rate to microfilaraemia was very low (less than $2.5 \%$ ). Conversion to asymptomatic microfilaraemia was nearly absent. (Transactions of the Royal Society of Tropical Medicine and Hygiene 2001; 95: 408-9).

\section{Drug resistant tuberculosis}

According to the WHO, the global status of tuberculosis (TB) has now become an emergency. The strategy used by most countries to meet the threat of TB is the "directly observed treatment short course (DOTS)" directed through a national TB control program. Challenges to the problem of multi-drug resistant TB (MDR-TB) should also be channelled 
Primary drug resistance to $\mathrm{TB}$ is the result of transmission of a resistant strain to someone with no previous history of the disease. Acquired drug resistance is usually the result of inadequate therapy due to inappropriate regime, duration, non-compliance or interruption in supply of drugs. Infection with MDR-TB has a high mortality. Sputum remains positive for longer periods compared to cases where the organisms are sensitive to the drugs and there is a high risk of transmission to contacts. Cross infection with MDR-TB is more likely in institutions such as hospitals, hostels, schools and prisons. Dealing with MDR-TB is not just a matter of sequentially adding yet another drug to a failing regime. There is no place for tinkering with regimes, because the assumption is that MDR-TB is always due to failure of effective implementation of the national program. (Journal of Tropical Paediatrics 2000; 46: 320-1). It is vital that all TB patients are managed according to national policies.

\section{Management of urticaria}

Urticaria is common and its main feature is the appearance of transient pruritic wheals. When the duration is less than 6 weeks it is classified as acute, whereas those lasting longer are regarded as chronic. Pruritus is quite distressing and often worse in the evening. Management should begin with an attempt to find the type of urticaria. Physical factors such as pressure, exertion and increased body heat are well known causes of urticaria. The management of urticaria produced by physical factors is based on their identification and avoidance. In children urticaria occurs with foods such as nuts, eggs, milk and sea food. Antimicrobials (especially the penicillins). NSAIDs and ACE inhibitors can cause urticaria. The drug treatment is based on use of histamine HI receptor antagonists. Both the older sedating antihistamines and the newer non-sedating ones are useful in the management; the latter group is preferred. In chronic urticaria it is recommended to give antihistamines regularly rather than as interrupted courses. When symptoms are not well controlled $\mathrm{H} 2$ receptor antagonists such as cimetidine could be added. Doxepin, which is an antidepressant, also gives benefit to some patients with chronic urticaria. Although there is rapid symptomatic improvement with steroids they are not recommended for chronic use. Counselling of patients is essential in the management of chronic urticaria. (Australian Prescriber 2001; 24: 124-6). Sri Lankan people often complain of urticaria after eating pineapple, tomato, prawns or crab. In such cases these items should be avoided.

R L Jayakody, Senior Lecturer in Pharmacology, Faculty of Medicine, University of Colombo.

\section{Health and intellectual property rights}

First, the patent holder can exclude direct competition, and charge higher prices for patented medicines than would have prevailed in a competitive market. Life-saving drugs can thus be made unaffordable, as has been seen particularly dramatically in sub-Saharan Africa.

Second, most developing countries are excluded from the benefits of protection for inventions because they lack the scientific infrastructure and the capital needed for research and development. High costs and the need for economies of scale place the development of patentable pharmaceuticals beyond the reach of most of them. (Carlos M Correa. Bulletin of the World Health Organization 2001; 79: 381). 